Dies ist die vorletzte Version eines Aufsatzes der in der Zeitschrift für philosophische Forschung, Band 65 (2011) erscheint.

\title{
Antiskeptische Trittbrettfahrer des semantischen Externalismus
}

Jochen Briesen, Bonn

\section{Einleitung}

Skeptische Argumente versuchen zum Teil unter Rekurs auf sogenannte skeptische Hypothesen $\mathrm{zu}$ beweisen, dass wir (fast) nichts von der uns umgebenden Außenwelt wissen. Eine interessante und viel beachtete Reaktion auf diese Form der Skepsis basiert auf dem semantischen Externalismus. Aber schafft es der Externalismus wirklich, skeptische Argumente befriedigend zurückzuweisen? Oder ist er zumindest in der Lage, den Weg für weiterführende und letztlich erfolgreiche antiskeptische Folgestrategien zu ebnen?

Bevor diese Fragen beantwortet werden können, muss in Abschnitt 2 zunächst geklärt werden, welches skeptische Argument überhaupt in den Blick genommen wird und wie plausibel dessen Prämissen sind? Die Abschnitte 3 und 4 beschäftigen sich dann mit der antiskeptischen Strategie des semantischen Externalismus. Es handelt sich um eine Strategie, die auf Überlegungen Hilary Putnams zurückgeht und durch Olaf Müller ausführlich verteidigt und entscheidend verbessert wurde (s. Putnam 1981, Müller 2003). Anschließend wird in Abschnitt 5 nachgewiesen, dass das skeptische Argument durch eine kleine aber entscheidende Modifikation gegen den antiskeptischen Angriff des semantischen Externalismus immunisiert werden kann. Abschnitt 6 nimmt sich schließlich der Frage an, ob die umformulierte Fassung des skeptischen Arguments anfälliger für antiskeptische Angriffe ist als die ursprüngliche Version. Gibt es antiskeptische 
Trittbrettfahrer, welche die vom Externalismus erzwungene Modifikation des Arguments insofern ausnutzen, als sie antiskeptische Strategien entwickeln, die in Bezug auf das ursprüngliche Argument noch nicht zur Verfügung standen? Drei mögliche Trittbrettfahrer dieser Art werden untersucht und letztlich als unbefriedigend zurückgewiesen. Die antiskeptische Reichweite des Externalismus ist und bleibt beschränkt.

\section{Die philosophische Skepsis}

Es gibt eine große Bandbreite skeptischer Argumente, die scheinbar anhand plausiber Prämissen beweisen, dass unsere Überzeugungen hinsichtlich der uns umgebenden Wirklichkeit keine Fälle von Wissen darstellen. Eine Klasse dieser Argumente macht dabei essentiellen Gebrauch von sogenannten skeptischen Hypothesen. Die in der heutigen Diskussion gängige skeptische Hypothese ist das Gehirn-im-Tank Szenario $(\mathrm{SH})$.

(SH) Angenommen der Person X wurde gleich zu Beginn ihrer Existenz das Gehirn aus dem Schädel gelöst und von Außerirdischen in einem Tank mit entsprechender Nährlösung aufbewahrt. Die Erde, wie wir sie kennen, hat niemals existiert und der Tank befindet sich auf einem völlig öden Planeten. X' Gehirn wird außerdem durch Elektroden und einen Supercomputer auf eine Weise stimuliert, die den gesamten Erfahrungshaushalt von X simuliert. Diese Simulation ist so perfekt, dass das Gehirn-im-Tank kein introspektives Kriterium besitzt, zu erkennen, ob dessen Erfahrung auf einer Simulation beruht oder nicht. Nehmen wir an, wir seien in genau der Situation, in der sich $\mathrm{X}$ befindet.

Das in der gegenwärtigen Debatte vorrangig diskutierte skeptische Argument, das unter Rekurs auf SH zu beweisen sucht, dass wir (fast) nichts von der Außenwelt wissen, lautet:

(1) Wenn S weiß, dass p, dann weiß S, dass non-SH. 
(2) S weiß nicht, dass non-SH.

(3) Also: S weiß nicht, dass p.

In dem Argument steht »S« für ein epistemisches Subjekt, »non-SH« besagt, dass $\mathrm{SH}$ falsch ist bzw. dass die Hypothese $\mathrm{SH}$ nicht realisiert ist, und »p« steht für eine Proposition, die von der uns umgebenden Wirklicheit handelt, wie beispielsweise »Vor mir liegt ein Bleistift $\ll$.

Das Argument ist logisch gültig und seine Prämissen sind zumindest auf den ersten Blick plausibel. Prämisse (1) ist plausibel, weil sie auf dem weithin akzeptierten Prinzip beruht, dass Wissen unter gewusster logischer Implikation geschlossen ist. Dieses Geschlossenheitsprinzip besagt:

Wenn S weiß, dass $\phi$, und S weiß, dass $\phi \gamma$ impliziert, dann weiß S, dass $\gamma$.

Weil p non-SH impliziert und weil dieses Implikationsverhältnis leicht einzusehen ist - es folgt allein aus der Formulierung von SH -, kann Prämisse (1) als verkürzte Instanz dieses Prinzips verstanden werden. Solange man also das Geschlossenheitsprinzip akzeptiert, ist Prämisse (1) überaus plausibel. ${ }^{1}$

Ein naheliegender Weg Prämisse (2) zu plausibilisieren, besteht in dem Hinweis darauf, dass $\mathrm{S}$ weder a posteriori noch a priori weiß, dass non-SH. Warum weiß S nicht a posteriori, dass non-SH? Aposteriorisches Wissen zeichnet sich dadurch aus, dass empirische Gründe für den vermeintlichen Fall von Wissen sprechen. Dies ist jedoch in Bezug auf die Überzeugung »non-SH« nicht der Fall. Person S kann nicht durch empirische Gründe ausschließen, dass sie ein Gehirn-im-Tank ist, dessen Erfahrungshaushalt nur simuliert wird. Denn jeder empirische Grund, der dieses Szenario ausschließen und somit die Überzeugung »non-SH« rechtfertigen könnte, könnte selbst Teil der Simulation sein. Die Überzeugung »non-SH« ist also nicht durch empirische

\footnotetext{
1 John Hawthorne (2003) hat in seiner Verteidigung des Geschlossenheitsprinzips darauf aufmerksam gemacht, dass das Prinzip nur in einer umformulierten und etwas schwächeren Fassung verteidigt werden kann. Falls dies richtig ist, so müssten die skeptischen Prämissen (1) und (2) dieser umformulierten Version des Prinzips angepasst werden.
} 
Gründe gerechtfertigt, daher kann S nicht a posteriori wissen, dass non-SH. ${ }^{2}$

Warum weiß S nicht a priori, dass non-SH? Apriorisches Wissen zeichnet sich dadurch aus, dass der vermeintliche Fall von Wissen durch nicht-empirische Gründe gerechtfertigt ist. Die Überzeugung »non-SH» macht aber eine Aussage über die Welt bzw. über die Position eines Subjekts in der Welt. Und um eine Überzeugung, die sich in dieser Weise auf die Welt bezieht, zu rechtfertigen, braucht man empirische Gründe - ich muss in der Welt nachschauen, um meine Position in ihr bestimmen zu können. Die Überzeugung »non-SH« ist folglich nicht durch nicht-empirische Gründe gerechtfertigt, und daher weiß S nicht a priori, dass non-SH. Die Überlegung, die Prämisse (2) plausibel macht, lässt sich also folgendermaßen zusammenfassen: (a) S kann nicht a posteriori wissen, dass non-SH. (b) S kann nicht a priori wissen, dass non-SH. (c) Alle Fälle von Wissen, die nicht a posteriori sind, sind Fälle apriorischen Wissens. Also folgt: (d) S weiß nicht, dass non-SH.

Das skeptische Argument beweist also anhand prima facie plausibler Prämissen, dass (fast) keine Überzeugung bezüglich der uns umgebenden Außenwelt ein Fall von Wissen ist. Weil diese Konklusion in den Augen vieler Philosophen unhaltbar ist, gibt es zahlreiche Versuche, das Argument zu blockieren. Der folgende Abschnitt widmet sich dem entsprechenden Versuch des semantischen Externalismus.

\section{Die antiskeptische Strategie des semantischen Externalismus}

Die antiskeptische Strategie des semantischen Externalismus lässt sich am besten als eine Strategie verstehen, die das skeptische Argument blockiert, indem sie Prämisse (2) zurückweist. Prämisse (2) wurde im vorangegangenen Abschnitt durch den Verweis darauf plausibilisiert, dass non-SH weder a posteriori noch a priori gewusst wird.

\footnotetext{
${ }^{2}$ Obwohl der angeführte Gedankengang sicherlich nicht unproblematisch ist, möchte ich ihn im Laufe dieses Textes akzeptieren. Zwei prominente Wege, ihn zu hinterfragen wären: (a) Der epistemische Externalismus wie in beispielsweise Timothy Williamsons (2000) vertritt. Und (b) der von James Pryor (2000) vertretene Dogmatismus. Auf die antiskeptischen Strategien (a) und (b) wird hier nicht eingegangen. Der vorliegende Text wird sich ganz auf die Strategie des semantischen Externalismus und möglicher Trittbrettfahrer konzentrieren.
} 
Demgegenüber vertreten einige Externalisten die These, dass man sehr wohl in der Lage ist a priori zu wissen, dass non-SH bzw. dass man kein Gehirn-im-Tank ist. Sie glauben, dass bestimmte semantische Überlegungen, die man aus dem philosophischen Lehnstuhl heraus anstellen kann, beweisen, dass wir nicht mit dem Gehirn-im-Tank aus SH identisch sein können.

Es gibt verschiedene Vorschläge, wie der semantische Externalismus sein antiskeptisches Beweisziel erreichen könnte. Ein übersichtliches Argument, an dem sowohl die Kernthesen des Externalismus als auch die Probleme seiner antiskeptischen Strategie übersichtlich illustriert werden können, lautet wie folgt: ${ }^{3}$

(4) Der Ausdruck »Tiger« eines Gehirn-im-Tanks bezieht sich nicht auf Tiger.

(5) Mein Ausdruck »Tiger« bezieht sich auf Tiger.

(6) Also: Ich bin kein Gehirn-im-Tank.

Weil dieses Argument logisch gültig ist und prinzipiell von jedem epistemischen Subjekt S wiederholt werden kann, folgt aus (6) auch, dass S weiß, dass non-SH. Damit wäre dann das skeptische Argument an Prämisse (2) blockiert. Ob diese Blockade jedoch in antiskeptischer Hinsicht befriedigen kann, hängt unter anderem davon $a b$, inwiefern sich die Prämissen (4) und (5) rechtfertigen lassen. Die folgende Erläuterung von (4) wird in die Grundzüge der externalistischen Semantik einführen. Die Ausführungen zu (5) hingegen werden dann wichtige Überlegungen zum apriorischen Status des Arguments enthalten und so auch auf dessen größte Schwierigkeit hinweisen.

Prämisse (4) beruht gänzlich auf den Kernthesen des semantischen Externalismus. Ausgehend von dem Zwillingserden-Gedankenexperiment kommt Putnam (1975) zu einem negativ und einem positiv formulierten Schluss, die ich hier als Kernthesen des Externalismus verstehen möchte. ${ }^{4}$

3 Ich beschränke mich auf die Darstellung der antiskeptischen Strategie, die von den semantischexernalistischen Überlegungen Hilary Putnams (1975; 1981) ihren Ausgang nehmen. Auch die externalistischen Reflexionen Donald Davidsons haben unter einer bestimmten Lesart einen antiskeptischen Zug, auf den ich hier aber nicht näher eingehen kann.

${ }^{4}$ Die Thesen stehen in einer engen Beziehung zueianander. In logischer Hinsicht lässt sich beispielsweise festhalten, dass These 1 von These 2 impliziert wird. Trotzdem ist es im Rahmen 
These 1: Der Bezug von Artbegriffen ist nicht durch die internen Zustände des Subjekts bestimmt. (Unter einem internen Zustand des Subjekts kann man sowohl die physikalischen als auch die engen mentalen Zustände des Subjekts verstehen. Zusammen mit der These, dass Bedeutungen den Bezug eines Terms festlegen, kommt es zu dem bekannten Slogan: »meanings just ain't in the head!« (s. Putnam 1975, 227).)

These 2: Erfolgreiches Bezugnehmen benötigt angemessenen kausalen Kontakt zum Bezuggenommenen. (Das angesprochene Gedankenexperiment soll ergeben, dass der Bezug eines Artbegriffs nicht alleine von den internen Zuständen des Subjekts abhängt, sondern auch von der tatsächlichen Umwelt der Sprecher. Der kausale Kontakt der Sprecher mit dieser Umwelt ist daher notwendig für erfolgreiches Bezugnehmen.)

Mit Hilfe der Thesen 1 und 2 lässt sich Prämisse (4) motivieren: Das Gehirn-im-Tank gleicht mir zwar bezüglich der internen Eigenschaften - in unseren Köpfen spielen sich die gleichen neuronalen und engen psychologischen Vorgänge ab - das heißt aber nicht, dass wir uns auf die gleichen Dinge beziehen können (s. These 1). Um sich erfolgreich auf Exemplare einer natürlichen Art beziehen zu können, muss man in einem angemessenen Kausalverhältnis zu ihnen stehen (s. These 2). Das Gehirn-im-Tank unterhält aber zur Außenwelt keine angemessene kausale Verbindung, schließlich wird dessen gesamter Erfahrungshaushalt von dem Programm eines gigantischen Computers generiert. Deshalb kann sich ein Gehirn-im-Tank mit seinem Ausdruck »Tiger« auch nicht auf Tiger beziehen. Falls sich ein Gehirn-im-Tank mit dem Ausdruck »Tiger« überhaupt auf etwas beziehen kann, so wird es sich mit dem fraglichen Ausdruck vermutlich lediglich auf bestimmte binäre Konfigurationen im Simulationsprogramm des Computers beziehen. Man kann auch sagen, dass das Gehirn-im-Tank eine andere Sprache spricht als ich (z.B. Tank-Deutsch im Gegensatz zu Deutsch). Denn dessen Tiger-Ausdrücke (sowie die allermeisten seiner Wörter) beziehen sich nicht auf Dinge aus Fleisch und Blut, sondern

einer Erkläuterung der antiskeptischen Strategie des semantischen Externalismus hilfreich, beide Thesen gesondert anzuführen. 
(wenn überhaupt) auf Bit-Konfigurationen - sozusagen auf Bit-Tiger.

Wenn man also die externalistischen Kernthesen akzeptiert, kann Prämisse (4) Der Ausdruck »Tiger« eines Gehirn-im-Tanks bezieht sich nicht auf Tiger gerechtfertigt werden. Ohne die externalistischen Thesen hier detailliert diskutieren zu können, akzeptiere ich im Folgenden die externalistische Rechtfertigung von Prämisse (4) und stimme außerdem der Auffassung zu, dass diese Prämisse a priori gerechtfertigt ist, weil ihre Rechtfertigung auf philosophischen Gedankenexperimenten beruht und keinerlei empirische Untersuchung umfasst.

Wie steht es um Prämisse (5) Mein Ausdruck »Tiger« bezieht sich auf Tiger? Ein naheliegender Einwand lautet: Wenn (5) wahr sein soll, dann müssen Tiger existieren, die die Referenten meiner Ausdrücke darstellen. Wenn es keine Tiger gibt, dann kann mein Ausdruck »Tiger« auch auf keine Tiger Bezug nehmen. Um also zu rechtfertigen, dass sich »Tiger« auf Tiger bezieht, muss ich davon ausgehen, dass Tiger existieren. Durch die Existenzpräsupposition in (5) ist das betrachtete antiskeptische Argument allerdings sowohl epistemisch zirkulär als auch question-begging und damit unbefriedigend. ${ }^{5}$

Ein Argument ist epistemisch zirkulär, wenn die Rechtfertigung einer Prämisse des Arguments bereits die Richtigkeit der Konklusion voraussetzt. Und um Prämisse (5) zu rechtfertigen, muss die Richtigkeit der Konklusion des antiskeptischen Arguments bereits vorausgesetzt werden. Ich muss annehmen, dass ich kein Gehirn-im-Tank in einem tigerlosen Universum bin, um zu rechtfertigen, dass sich mein Ausdruck »Tiger« auf Tiger bezieht. Außerdem muss jede Rechtfertigung von (5) question-begging gegenüber dem Skeptiker sein. Denn jede Rechtfertigung von (5) muss voraussetzen, dass es Tiger gibt und macht damit Gebrauch von Außenweltwissen, das wir in unserer momentanen

\footnotetext{
${ }^{5}$ Es hilft aus meiner Sicht nicht, der Existenzpräsupposition dadurch entgehen zu wollen, dass man die Prämissen des Arguments konditionalisiert: (5k) Falls es Tiger gibt, dann bezieht sich mein Ausdruck »Tiger " auf Tiger; (4k) Falls es Tiger gibt, dann bezieht sich der Ausdruck "Tiger « eines Gehirn-im-Tanks nicht auf Tiger. Denn es ist zumindest unklar, ob anhand von (5k) und (4k) noch auf die gewünschte Konklusion (6) geschlossen werden kann. Um anhand der fraglichen Prämissen auf einen tatsächlichen Unterschied zwischen mir und dem Gehirn-im-Tank schließen zu können, muss ich voraussetzen, dass es Tiger gibt. Vgl. hierzu auch Müller (2003, 167ff).
} 
dialektischen Situation gegen den Skeptiker gerade nicht anbringen dürfen. ${ }^{6}$

\section{Verbesserung der externalistischen Strategie gegen die Skepsis}

Der folgende Vorschlag, dem eben formulierten Problem zu entgehen, geht auf Olaf Müller zurück (Müller 2003, 167-183). ${ }^{7}$ Die Idee besteht in einer zweifachen Modifikation des antiskeptischen Ausgangsarguments. Dem Argument wird erstens eine disjunktive Prämisse hinzugefügt. Und zweitens wird jedes Vorkommnis von »Tiger« durch »Gehirn« ersetzt:

(o) Entweder es gibt Gehirne oder es gibt keine Gehirne.

Fall I: Es gibt Gehirne.

(4)* Der Ausdruck »Gehirn« eines Gehirn-im-Tanks bezieht sich nicht auf Gehirne.

(5)* Mein Ausdruck »Gehirn« bezieht sich auf Gehirne.

(6)* Also bin ich kein Gehirn-im-Tank.

Fall II: Es gibt keine Gehirne.

(7)* Also bin ich kein Gehirn-im-Tank.

Die Prämisse (o) kann a priori gerechtfertigt werden, denn sie beruht auf dem logischen Prinzip des ausgeschlossenen Dritten. Prämisse (4)* kann auf die gleiche Weise gerechtfertigt werden, wie oben Prämisse (4) gerechtfertigt wurde, solange man zugesteht, dass »Gehirn« ein Begriff ist, auf den die externalistischen Kernthesen ebenso zutreffen

\footnotetext{
${ }^{6}$ Im Gegensatz zur epistemischen Zirkularität ist die Eigenschaft Question-begging-Sein eine relationale Eigenschaft eines Arguments A zu einem Argument B. Letztere Eigenschaft ist im Gegensatz zur ersten also abhängig von der dialektischen Situation, in die ein Argument eingebettet ist.

7 Allerdings formuliert Müller die angegebene Verbesserung etwas anders. Dies hat zwei Gründe: Erstens kommt Müller auf anderen Pfaden zu seinem Verbesserungsvorschlag und zweitens soll seine verbesserte Fassung zwar auf das gleiche Problem reagieren, allerdings taucht das Problem bei ihm an einer anderen Stelle auf.
} 
wie auf »Tiger«. ${ }^{8}$ Die Prämisse (5)* ist unter Fall I nun auch nicht mehr problematisch. Denn unter der Voraussetzung, dass es Gehirne gibt, ergibt sich Prämisse (5)* allein aufgrund der Funktion und Verwendungsweise der Anführungsstriche. (5)* kann also unter Fall I insofern a priori gerechtfertigt werden, als zu dessen Rechtfertigung nur sprachliches Wissen und kein empirisches Außenweltwissen in Anschlag gebracht werden muss. ${ }^{9}$ Aus (4)* und (5)* folgt dann die Konklusion (6)*, die besagt, dass ich kein Gehirn-im-Tank bin. Und die Konklusion (7)*, die dasselbe besagt, ergibt sich alleine aus der Beschreibung des Falles II. Mit diesem Beweis liegt nun tatsächlich ein schlagkräftiges Argument gegen den Skeptizismus vor. Das Argument ist weder epistemisch zirkulär noch question-begging und es beweist schlüssig, dass die skeptische Hypothese SH falsch ist. Weil diese antiskeptische Überlegunge prinzipiell von jeder Person S nachvollzogen werden kann, ist damit auch die skeptische Prämisse (2) $S$ weiß nicht, dass non-SH widerlegt und das angeführte skeptische Argument ist blockiert.

Einen letzten Einwand gegen die vorgestellte antiskeptische Strategie des Externalismus gilt es noch auszuräumen. Der Einwand besteht in dem Hinweis darauf, dass der antiskeptische Beweis auch von einem Gehirn-im-Tank nachvollzogen werden könnte. Dieses Gehirn würde jeden Schritt des Beweises ebenso nachvollziehen können wie wir und es würde deswegen ebenfalls zu dem Schluss kommen: Ich bin kein Gehirnim-Tank. Allerdings wäre dieser Schluss falsch, denn er würde ja von einem Gehirn gezogen, das tatsächlich im Tank steckt. In dem antiskeptischen Argument muss also ein Fehler liegen.

Dieser Vorwurf ist schnell ausgeräumt, wenn man externalistische Thesen

\footnotetext{
${ }^{8}$ Jede Person, die daran zweifelt, dass der Begriff »Gehirn« ebenso externalistischen Thesen unterworfen ist wie beispielsweise der Begriff »Tiger«, weil »Gehirn« im Gegensatz zu »Tiger« kein natürlicher Artbegriff ist, ersetze alle Vorkommnisse des Ausdrucks »Gehirn« im vorliegenden Text durch den Term "menschliches Gehirn«, der offensichtlich ein natürlicher Artbegriff ist. An den hier vorgebrachten Überlegungen ändert das nichts. Außerdem sei in diesem Zusammenhang auf Müllers Überlegungen verwiesen, warum das Argument letztlich gar nicht davon abhängt, ob »Gehirn« ein Artbegriff ist oder nicht (s. Müller 2003, 190-198).

${ }^{9}$ Dass (5)* unabhängig von Weltwissen ist, kann durch folgende Formulierung der Prämisse verdeutlicht werden: Mein Begriff "Gehirn « bezieht sich auf Gehirne (was auch immer das ist). Wer allerdings sprachliches Wissen bezüglich der Bedeutung von Anführungsstrichen schon als empirisches Wissen auffassen möchte, dem sei dies zugestanden. Jedoch sei darauf verwiesen, dass es sich dabei um eine Form von Weltwissen handelt, das vom skeptischen Argument nicht angegriffen wird. Daher ist das Anbringen dieses sprachlichen Wissens bei der Rechtfertigung von (5)* weder zirkulär noch question-begging.
} 
akzeptiert. Es ist gezeigt worden, dass das Gehirn-im-Tank eine andere Sprache spricht als wir. Damit müssten wir also den antiskeptischen Beweis, wenn er von einem Gehirn-imTank nachvollzogen wird, in dessen Sprache übersetzen. In Tank-Deutsch lautet die Konklusion des Beweises etwa: Ich bin kein Bit-Gehirn-in-einem-Bit-Tank. Dieser Satz ist aber auch aus dem Munde eines echten Gehirn-im-Tanks wahr. Das eben formulierte Problem ist damit ausgeräumt und der antiskeptische Beweis rehabilitiert. Der Beweis zeigt schlüssig, dass ein Subjekt nicht das sein kann, was es in seiner Sprache mit dem Ausdruck »Gehirn-im-Tank« bezeichnet. Dieses Ergebnis genügt, um das skeptische Argument zu blockieren.

\section{Der skeptische Rückzug}

Die verbesserte antiskeptische Beweisführung des Externalismus enthält keine offensichtlichen Fehler und blockiert erfolgreich das skeptische Argument. Dem Skeptiker bleibt nur die Möglichkeit, sein ursprüngliches Argument umzuformulieren, so dass es gegen die antiskeptische Blockade immun ist. Die Blockade beruht auf dem Nachweis, dass die skeptische Hypothese SH falsch ist. Dieser Nachweis beruht seinerseits auf der externalistischen Einsicht, dass sich die Begriffe eines Gehirn-im-Tanks nicht auf die Dinge beziehen, auf die sich unsere Begriffe beziehen. Der Skeptiker muss daher versuchen, in Übereinstimmung mit den externalistischen Kernthesen die skeptische Hypothese so umzugestalten, dass das hypothetische Gehirn-im-Tank auf die gleichen Dinge Bezug nehmen kann wie wir. Und er muss sein Argument dieser umgestalteten Hypothese anpassen.

Eine skeptische Hypothese SH2, die eben genannte Anforderungen erfüllt, ist schnell gefunden:

(SH2) Diese Hypothese gleicht der ursprünglichen skeptischen Hypothese SH bis auf zwei kleine aber entscheidende Punkte: Das Gehirn der Person X ist erst vor fünf Minuten eingetankt worden und die Erde wurde erst danach zerstört. 
Warum kann sich ein vor fünf Minuten eingetanktes Gehirn auch im Angesicht externalistischer Kernthesen auf die gleichen Dinge beziehen wie wir? Dies ist möglich, weil die externalistischen Thesen gar nicht ausschließen, dass ein kürzlich eingetanktes Gehirn mit den Ausdrücken »Tiger«, »Gehirn«, usw. auf Tiger und Gehirne Bezug nimmt. Dem Externalismus zufolge kann sich ein seit Lebzeiten eingetanktes Gehirn deswegen nicht auf Tiger usw. beziehen, weil sich dessen Kausalgeschichte grundlegend von der unseren unterscheidet - das Gehirn-im-Tank durfte nie angemessenen kausalen Kontakt zu Tigern usw. unterhalten. Eben dies ist bei kürzlich eingetankten Gehirnen aber nicht der Fall. Ein Gehirn-im-Tank, das erst vor fünf Minuten eingetankt wurde, konnte in der Zeit vor seiner Eintankung prinzipiell zu den gleichen Dingen kausale Verbindungen unterhalten wie Du und ich. Zumindest in der ersten Zeit seiner Eintankphase wird sich daher ein solches Gehirn-im-Tank mit seinen Ausdrücken auf die gleichen Dinge beziehen wie wir. Und dessen Aussagen der Form »Vor mir steht ein ausgewachsener Tiger« wären deswegen auch alle falsch. Der letzte Term dieses Satzes bezöge sich nämlich auf Tiger und in der tatsächlichen Umgebung des kürzlich eingetankten Gehirns befänden sich keinerlei Tiger, sondern nur noch Bit-Tiger. ${ }^{10}$

Wenn das skeptische Argument gegen die externalistische Blockade immun sein will, muss es die ursprüngliche skeptische Hypothese durch SH2 ersetzen:

(1)* Wenn S weiß, dass $\mathrm{p}$, dann weiß S auch, dass non-SH2.

(2)* S weiß nicht, dass non-SH2.

(3)* Also: S weiß nicht, dass p.

Beide Prämissen (1)* und (2)* können (zumindest auf den ersten Blick) auf die gleiche

${ }^{10}$ Der Fall eines kürzlich eingetankten Gehirns ist in semantischer Hinsicht mit einer Reise von unserer Erde zur Putnamschen Zwillingserde vergleichbar, auf der Wasser nicht $\mathrm{H}_{2} \mathrm{O}$, sondern XYZ ist. Paul Boghossian (1989) geht in seinem slow-switching Argument davon aus, dass eine Person, die unbemerkt von der Erde zur Zwillingserde gebracht würde, ihre Sprache langsam der Zwillingserdenumgebung anpassen würde. Auch Boghossian macht aber deutlich, dass dieser Sprachwandel eine gewisse Zeit bräuchte. Über diesen Punkt scheint in der philosophischen Literatur Einigkeit zu herrschen. Auch ich gestehe zu, dass sich die Bezugnahme des kürzlich eingetankten Gehirns im Laufe der Zeit verändern wird, allerdings setzt diese Veränderung sicherlich nicht plötzlich ein. 
Weise gerechtfertigt werden, wie oben die ursprünglichen skeptischen Prämissen (1) und (2) gerechtfertigt wurden (s. Abschnitt 2). Allerdings kann der Externalismus die Rechtfertigung von (2)* im Gegensatz zur Rechtfertigung von (2) nicht mehr durch semantische Überlegungen zurückweisen. Weil das Argument außerdem durch die Modifikation natürlich seine logische Gültigkeit nicht einbüßt, beweist es auch im Angesicht des Externalismus schlüssig, dass wir über (fast) keinerlei Außenweltwissen verfügen. Die antiskeptische Durchschlagskraft des Externalismus ist daher beschränkt. Es ist möglich, das skeptische Argument durch eine naheliegende Modifikation gegen den externalistischen Angriff zu immunisieren.

Auf einen Unterschied zwischen dem ursprünglichen und dem vom Externalismus erzwungenen skeptischen Argument muss allerdings in aller Deutlichkeit hingewiesen werden: Die Reichweite des angezweifelten Wissens ist im umformulierten Argument geringer. Dies liegt daran, dass Prämisse (1)* durch das Geschlossenheitsprinzip gerechtfertigt ist und diese Rechtfertigung voraussetzt, dass non-SH2 von $\mathrm{p}$ impliziert wird (s. Abschnitt 2). Nur für Propositionen, die die Falschheit von SH2 implizieren, kann das skeptische Argument beweisen, dass wir in keiner Wissensrelation zu ihnen stehen.

Dieser Punkt soll durch ein Beispiel veranschaulicht werden:

x. Bei meinem Zoobesuch vor einer Woche konnte ich einen Tiger beobachten, der von seinem Wärter gestreichelt wurde.

Die Überzeugung, dass x, impliziert zwar non-SH aber sie impliziert nicht non-SH2. Auch wenn ich tatsächlich ein seit fünf Minuten eingetanktes Gehirn wäre, könnte x durchaus wahr sein. Daher kann x nicht in das umformulierte Argument eingesetzt werden. Die Prämisse (1x)* Wenn $S$ weiß, dass $x$, dann weiß $S$ auch, dass non-SH2 ist schlicht und einfach falsch.

Die allgemeine Lehre, die aus diesem Beispiel gezogen werden kann, lautet: Für den Bereich unserer Außenweltüberzeugungen, der sich auf die Vergangenheit bezieht, kann der Skeptiker anhand seines umformulierten Arguments nicht mehr beweisen, dass es keine Fälle von Wissen sind. Der Frage, ob diese Einschränkung dem Skeptiker 
schließlich zum Verhängnis wird, wird in den nächsten Abschnitten nachgegangen.

\section{Antiskeptische Trittbrettfahrer des Semantischen Externalismus}

Der Externalismus zwingt den Skeptiker, die ursprüngliche skeptische Hypothese SH durch die Hypothese des kürzlich eingetankten Gehirns SH2 zu ersetzen. Gegen das auf diese Weise umformulierte Argument können externalistische Thesen nichts mehr ausrichten. Ist aber das skeptische Argument durch das erzwungene Einsetzen von SH2 in einer anderen Hinsicht geschwächt worden? Oder kann gerade die Möglichkeit, ein kürzlich eingetanktes Gehirn zu sein, auf bisher ungeahnte Weise ausgeschlossen werden? Meiner Meinung nach gibt es diesbezüglich drei Ausschlussversuche.

\subsection{Erster Versuch: Modifikation und Bedrohung}

Der erste Folgeangriff auf das modifizierte skeptische Argument lautet:

Das modifizierte Argument ist durch $\mathrm{SH} 2$ in seiner Reichweite soweit eingeschränkt, dass es keine echte Bedrohung mehr darstellt. Deswegen bedarf es gar keiner substanziellen Lösung mehr.

Dieser Folgeangriff ist wenig überzeugend. Die Reichweite eines skeptischen Arguments hat nur wenig mit dessen Lösungsbedürftigkeit zu tun hat. Der Skeptiker generiert bereits dann ein bedrohliches und lösungsbedürftiges Problem, wenn er für einen Teil unserer vermeintlichen Paradefälle von Außenweltwissen, schlüssig beweist, dass es keine Fälle von Wissen sind. Wie groß dieser Teil ist, ist zweitrangig. Damit bleibt nur noch die Frage, ob das modifizierte skeptische Argument Paradefälle von Außenweltwissen in Zweifel ziehen kann. Diese Frage muss sicherlich bejaht werden. Das modifizierte Argument ist zwar insofern eingeschränkt, als es unser Außenweltwissen bezüglich der 
Vergangenheit nicht mehr bezweifelt. Unser Wissen, das sich auf unsere gegenwärtige Umgebung bezieht, ist von der Modifikation jedoch nicht berührt. Gerade die Überzeugungen, die sich auf mittelgroße Gegenstände in unserer unmittelbaren und gegenwärtigen Umgebung beziehen, scheinen allerdings besonders geeignete Kandidaten für Paradefälle von Außenweltwissen zu sein. Und für Überzeugungen dieser Art kann das skeptische Argument schlüssig beweisen, dass sie kein Fall von Wissen sind - das ist problematisch genug.

\subsection{Zweiter Versuch: Erinnerungen}

Der zweite Folgeangriff auf das modifizierte skeptische Argument lautet:

Das umformulierte skeptische Argument kann für unsere Außenweltüberzeugungen bezüglich der Vergangenheit nicht beweisen, dass es keine Fälle von Wissen sind. Diese Überzeugungen können daher angeführt werden, um die skeptische Prämisse $(2)^{*} \mathrm{zu}$ widerlegen. Unser Vergangenheitswissen kann ausschließen, dass wir ein kürzlich eingetanktes Gehirn sind. Damit ist das skeptische Argument blockiert.

Manche der Außenweltüberzeugungen, die sich auf meine Vergangenheit beziehen oder die ich in der Vergangenheit erworben habe, sind empirische Gründe, die a posteriori rechtfertigen, dass ich kein vor fünf Minuten eingetanktes Gehirn bin. Ein Beispiel wäre die gestrig erworbene Überzeugung, dass die Medizin noch Jahre der Forschung vor sich haben wird, um ein Gehirn in einem Tank überhaupt am Leben zu erhalten. Gründe dieser Art haben in Bezug auf non-SH2 rechtfertigende Kraft, weil sie nicht auf der Simulation beruhen können, von der in SH2 die Rede ist - schließlich bin ich laut SH2 erst seit fünf Minuten eingetankt, meine Erinnerungen beruhen daher sicherlich nicht auf der Simulation von der in SH2 die Rede ist. Weil das modifizierte Argument des Skeptikers außerdem für die eben formulierte Beispielüberzeugung nicht beweisen kann, dass es kein Fall von Wissen ist, ist die empirische Rechtfertigung von non-SH2 auch nicht question- 
begging gegenüber dem Skeptiker: SH2 kann ausgeschlossen werden, ohne etwas vorauszusetzen, das der Skeptiker bezweifelt. Die Prämisse (2)* $S$ weiß nicht, dass nonSH2 ist also a posteriori widerlegbar und der Skeptiker steckt in einem Dilemma. Entweder er formuliert sein Argument mit der Hypothese $\mathrm{SH}$, dann kann er nicht a posteriori aber unter Rekurs auf externalistische Kernthesen a priori widerlegt werden. Oder er formuliert das Argument mit SH2 und kann nicht a priori aber a posteriori widerlegt werden. Das ist ein vortrefflich antiskeptisches Ergebnis.

Leider sind die zu diesem Ergebnis führenden Überlegungen fehlerhaft. Es stimmt zwar, dass die eben genannte Strategie nicht question-begging ist. Die bei der empirischen Rechtfertigung verwendeten Erinnerungen unterstehen keinem skeptischen Zweifel, d.h. Überzeugungen, die auf diesen Erinnerungen beruhen, können nicht erfolgreich in das skeptische Argument eingesetzt werden. Daraus folgt aber nicht, dass die angedeuteten empirischen Gründe nicht trotzdem auf der Simulation beruhen können, von der in SH2 die Rede ist. Es ist durchaus vorstellbar, dass die Erfahrungssimulation - auch wenn sie erst vor fünf Minuten begonnen hat - sowohl meine gegenwärtigen Erfahrungen als auch meine Erinnerungen simuliert. Wenn aber auch die Dinge in meinem Bewusstsein, die sich auf die Vergangenheit beziehen, auf der erst kürzlich eingetretenen Simulation beruhen können, dann kann non-SH2 nicht durch Überzeugungen bezüglich der Vergangenheit, gerechtfertigt werden. Diese Überzeugungen hätten - wie im Fall non-SH - keine rechtfertigende Kraft, die Simulationshypothese auszuschließen, weil sie genau auf der Simulation beruhen könnten, die sie auszuschließen gedenken.

Spricht irgendetwas gegen die Möglichkeit, dass anhand der Hypothese SH2 auch unsere Erinnerungen simuliert sein könnten? Ich denke nicht. Die Möglichkeit der Erinnerungssimulation kann in unserer dialektischen Situation auf zweierlei Weise plausibel gemacht werden. Erstens, wer akzeptiert, dass Medizin, Neurologie und computergestützte Simulationstechnik zusammen prinzipiell in der Lage sind, alle gegenwärtigen Erfahrungen einer Person täuschend echt $\mathrm{zu}$ simulieren, scheint die Möglichkeit, dass auch bestimmte Erinnerungen dieser Person durch die gleiche Methode simuliert werden können, nicht mehr prinzipiell ausschließen zu können. Zweitens, ein Traum ist in gewisser Hinsicht mit der Hypothese SH2 vergleichbar. Angenommen mein 
Traum hat vor fünf Minuten begonnen. So kann ich träumen, vor einem Apfel zu sitzen und aufgrund dessen die Überzeugung unterhalten, dass vor mir ein Apfel liegt, ohne dass tatsächlich ein Apfel in meiner näheren Umgebung ist. Ich kann aber ebenso gut träumen, gestern ein informatives Gespräch über die Überlebenschancen eines eingetankten Gehirns mit einer Reihe von Medizinern geführt zu haben und die entsprechende Überzeugung unterhalten, ohne dass ein solches Gespräch tatsächlich stattgefunden hätte. Obwohl ich also erst seit kurzem träume, ist es durchaus möglich, dass auch die Dinge in meinem Bewusstsein, die sich auf die Vergangenheit beziehen, auf dem Traum basieren. Wenn ein Traum in der Lage ist, meine Erinnerungen sozusagen zu überschreiben, warum sollte dann eine Computersimulation dazu nicht in der Lage sein?

Zusammenfassend lässt sich sagen: Obwohl das modifizierte skeptische Argument für Überzeugungen bezüglich der Vergangenheit nicht beweist, dass es keine Fälle von Wissen sind, kann die modifizierte skeptische Hypothese SH2 anhand dieser Überzeugungen nicht ausgeschlossen werden. Denn die Erinnerungen und die auf ihnen basierenden Überzeugungen könnten trotz allem auf der Simulation beruhen, von der in SH2 die Rede ist. Damit ist die auf Erinnerungen basierende antiskeptische Folgestrategie zurückgewiesen.

Doch gibt es nicht einen einfachen Weg, die fragliche Folgestrategie zu rehabilitieren? Wenn man annimmt, dass die skeptische Hypothese nicht nur gegenwärtige Erfahrungen, sondern auch Erinnerungen simuliert, dann handelt es sich bei der skeptischen Hypothese streng genommen nicht mehr um SH2, sondern um eine Erweiterung der Hypothese $\mathrm{SH} 2$ - nennen wir sie »SH2*«. Nun ist es zwar korrekt, dass SH2* nicht durch unsere Erinnerungen ausgeräumt werden kann, doch das ist auch nicht nötig, denn in Bezug auf $\mathrm{SH}^{*}$ greift der ursprüngliche antiskeptische Einwand des semantischen Externalismus wieder. Schließlich kann sich ein kürzlich eingetanktes Gehirn, dessen gesamter Erfahrungs- und Erinnerungshaushalt, d.h. auch dessen gesamte Lerngeschichte simuliert wird, gemäß dem Externalismus nicht auf die gleichen Dinge beziehen wie wir. Damit ist der antiskeptische Schulterschluss des semantischen Externalismus mit der oben angeführten Erinnerungsstrategie letztlich doch erfolgreich.

Aus meiner Sicht ist diese Verteidigung der Erinnerungsstrategie allerdings nicht 
überzeugend. Aus den externalistischen Thesen folgt keineswegs, dass sich ein Gehirn-imTank aus SH2* nicht auf die gleichen Dinge beziehen kann wie wir. Nach dem Externalismus verlangt erfolgreiches Bezugnehmen angemessenen kausalen Kontakt zum Bezuggenommenen. Doch ein kürzlich eingetanktes Gehirn aus SH2* kann (genau wie ein kürzlich eingetanktes Gehirn aus SH2) in der Zeit vor seiner Eintankung prinzipiell zu den gleichen Dingen kausale Kontakte unterhalten wie Du und ich (vgl. Abschnitt 5). Bei einem kürzlich eingetankten Gehirn aus $\mathrm{SH}_{2} *$ sind zwar auch einzelne Erinnerungen simuliert, die Kausalgeschichte des fraglichen Gehirns ist davon aber nicht betroffen. Und genau diese Kausalgeschichte ist entscheidend, bei der Frage, ob sich das fragliche Gehirn-im-Tank im Lichte des Externalismus auf die gleichen Dinge beziehen kann wie wir. Es bleibt also dabei: Die auf Erinnerungen basierende antiskeptische Folgestrategie hinsichtlich des vom Externalismus erzwungenen modifizierten skeptischen Arguments scheitert.

\subsection{Dritter Versuch: Schluss auf die beste Erklärung}

Der dritte und letzte Folgeangriff auf das modifizierte skeptische Argument, den ich diskutieren möchte, lautet:

Die Hypothese SH2 muss bei der Erklärung unserer sinnlichen Erfahrungen eine Doppelstrategie verfolgen. Das Zustandekommen unserer Erfahrung vor der Eintankung muss anders erklärt werden als das Zustandekommen der Erfahrungen, die wir nach der Eintankung machen. Es ist daher möglich, eine etwas in Vergessenheit geratene Strategie gegen die skeptische Hypothese und damit gegen Prämisse (2)* $S$ weiß nicht, dass non-SH2 anzuführen: Der Schluss auf die beste Erklärung.

Die generelle antiskeptische Überlegung, die auf dem Schluss auf die beste Erklärung fusst, lässt sich wie folgt charakterisieren: Es gibt verschiedene Kriterien für eine gute 
Erklärung eines Phänomens. Ein allgemein akzeptiertes Kriterium für eine gute Erklärung ist deren Einfachheit. Bei gleicher Erklärungskraft ist die beste Erklärung eines Phänomens die einfachste und jede Erklärung, die das gleiche Phänomen weniger einfach erklärt, kann ausgeschlossen werden. Ein guter Ausgangspunkt für die Erklärung des Zustandekommens unserer sinnlichen Erfahrungen besteht in dem Hinweis auf eine Außenwelt mit allerhand Gegenständen, die kausal auf unsere Sinne wirken. Allerdings ist die Art und Weise, wie die skeptische Hypothese das Zustandekommen sinnlicher Erfahrungen erklärt, völlig anders. Auch wenn wir dem Skeptiker zugestehen, dass die skeptische Hypothese hinsichtlich ihrer Erklärungskraft nicht schwächer ist als die Außenwelthypothese, so ist die Erklärung anhand der skeptischen Hypothese doch weniger einfach und daher kann die fragliche Hypothese ausgeschlossen und das skeptische Argument blockiert werden.

Es sind zwei Herausforderungen, die mit dieser generellen antiskeptischen Strategie verbunden sind:

(I) Inwiefern ist die Erklärung, die die skeptische Hypothese für unsere Sinneserfahrungen gibt, weniger einfach als die Standard-Erklärung?

(II) Warum ist es epistemisch gerechtfertigt, die einfachere Erklärung einer komplexeren vorzuziehen?

Der Grund, warum die antiskeptische Strategie des Schlusses auf die beste Erklärung mehr oder weniger in Vergessenheit geraten ist, liegt in der Schwierigkeit auf die beiden Fragen (I) und (II) angemessen zu reagieren. ${ }^{11}$ Doch eventuell eröffnen sich neue Wege (I) und (II) zu beantworten, wenn man sich statt auf die traditionelle skeptische Hypothese $\mathrm{SH}$, auf die vom Externalismus erzwungene Hypothese SH2 konzentriert?

$\mathrm{Zu}$ Herausforderung (I). Bisher ist auf die Frage (I) noch keine Antwort gegeben worden, die auf breite Akzeptanz gestoßen wäre. Der neue und hier zur Debatte stehende Vorschlag, der auf eine Bemerkung David Chalmers' (2005, 35-36) zurückgeht, lautet,

${ }^{11}$ Was nicht heißen soll, es gäbe keine interessanten Vorschläge in diese Richtung, siehe z.B. Vogel (1990). 
dass bezüglich der modifizierten Hypothese SH2 (im Gegensatz zu SH), sehr leicht deutlich gemacht werden kann, inwiefern die Erfahrungserklärung weniger einfach ist: Die modifizierte Hypothese SH2 muss - weil sie davon ausgeht, dass wir erst kürzlich eingetankt wurden - bei der Erklärung des Zustandekommens unserer Erfahrungen eine Doppelstrategie verfolgen. Das Zustandekommen unserer Erfahrung vor der Eintankung muss anders erklärt werden als das Zustandekommen der Erfahrungen, die wir nach der Eintankung machen. Damit ist die Erklärung in jedem Fall komplizierter als die StandardErklärung unserer sinnlichen Erfahrungen, die eine einfache Strategie verfolgen kann (ungefähr so: Erfahrungen kommen durch kausalen Kontakt der Gegenstände mit unseren Sinnen zustande).

Diesbezüglich drängt sich allerdings ein Einwand auf. Ist nicht auch unsere Standard-Erklärung auf einen Doppelmechanismus festgelegt? Der Begriff der Erfahrung muss innerhalb der hier anvisierten antiskeptischen Argumentation in einem weiten Sinn verstanden werden. Es darf nicht definitorisch aus dem Erfahrungsbegriff folgen, dass eine sinnliche Erfahrung ein Objekt in der Außenwelt haben muss. Nur dann kann davon gesprochen werden, dass das Gehirn-im-Tank überhaupt Erfahrungen macht, die es zu erklären gilt. Weil nach diesem weiten Erfahrungsbegriff auch Halluzinationen als Erfahrungen gelten und Halluzinationen ebenso im vortanklichen Bewusstsein vorkommen, ist es nicht richtig, dass die Außenwelthypothese eine einfache Strategie bei der Erklärung unserer Erfahrungen verfolgen kann. Denn Halluzinationen können im Gegensatz zu Standarderfahrungen gerade nicht durch den kausalen Kontakt der Gegenstände mit unseren Sinnesorganen erklärt werden. Auch wenn wir also an der Außenwelthypothese festhalten und annehmen, wir seien nicht seit fünf Minuten eingetankt, müssen wir bei der Erfahrungserklärung eine Doppelstrategie verfolgen. Die angeführte Reaktion auf Herausforderung (I) kann demnach nicht überzeugen.

Diesem Problem kann allerdings begegnet werden. Wenn es stimmt, dass wir innerhalb der angeführten antiskeptischen Überlegung einen weiten Erfahrungsbegriff voraussetzen müssen und wenn daraus tatsächlich folgt, dass wir auch unabhängig von skeptischen Hypothesen eine Doppelstrategie bei einer generellen Erfahrungserklärung verfolgen müssen, so besagt das nur, dass die Hypothese SH2 mindestens eine dreifache 
Strategie verfolgen muss. Die Hypothese SH2 muss eine dreifache Strategie verfolgen, weil sie alles erklären muss, was auch die Standard-Erklärung erklärt (Normalerfahrungen und Halluzinationen) und zusätzlich die Erfahrungen, die nach der Eintankung gemacht werden. Die skeptische Erklärung unserer Erfahrungen gemäß der skeptischen Hypothese ist also in jedem Fall komplexer als die Standard-Erklärung. Unter der Voraussetzung, dass der semantische Externalismus eine Modifikation der skeptischen Hypothese hin zu SH2 erzwingt, kann also der Herausforderung (I) des Schlusses auf die beste Erklärung begegnet werden.

Zu Herausforderung (II). Unter Rekurs auf das Prinzip der Einfachheit könnte auf die Herausforderung (II) reagiert werden:

Von zwei Erklärungen, die sich in sonst allen entscheidenden Merkmalen (Erklärungskraft, usw.) gleichen, ist die einfachere die bessere - sie ist besser, weil sie mit größerer Wahrscheinlichkeit wahr ist als die weniger einfache. Deswegen sind wir epistemisch gerechtfertigt, die weniger einfache auszuschließen. ${ }^{12}$

Doch ist es plausibel anzunehmen, dass die einfachere Erklärung auch wahrscheinlicher ist? Wie kann diese Annahme im Angesicht skeptischer Argumente verteidigt werden? Auch wenn eine aposteriorische Rechtfertigung dieser Annahme möglich sein sollte, so wird sie in unserer dialektischen Situation nicht weiterhelfen. Der antiskeptischen Reanimation des Schlusses auf die beste Erklärung wird dann im besten Fall das gleiche Schicksal widerfahren, wie dem unter 6.2 diskutierten Versuch, SH2 bzw. SH2* mit Hilfe empirischer Gründe (die auf der Erinnerung beruhen) auszuschließen. Und eine apriorische Rechtfertigung scheint schlicht unmöglich. Schließlich ist es eine empirische Tatsache (wenn es überhaupt eine ist), dass die einfacheren Erklärungen wahrscheinlicher wahr sind als die weniger einfachen. Daher scheint das Prinzip der Einfachheit eine

\footnotetext{
12 Ich stimme Peter Lipton $(1991,61)$ zu, dass eine Erklärung hinsichtlich zweier Aspekte die beste sein kann: Eine Erklärung kann die »loveliest« Erklärung sein, so dass ihr, vorausgesetzt sie ist korrekt, die größte Erklärungskraft zukommt bzw. so dass sie ein umfassenderes Verständnis des zu erklärenden Phänomens ermöglicht als eine andere Erklärung. Oder eine Erklärung kann die »likeliest« Erklärung sein, so dass sie mit größerer Wahrscheinlichkeit wahr ist bzw. besser gerechtfertigt ist. In diesem Sinne lautet das oben angeführte Prinzip der Einfachheit: Gegeben E1 und E2 sind gleich »lovely«, wenn E1 einfacher ist als E2, dann ist E1 »more likely to be true«.
} 
empirische These auszudrücken, die a priori nicht gerechtfertigt werden kann.

»Why, after all, should it be thought (...) that the world is more likely to be simple than complex?“" (BonJour 1998, 91)

Kann unter Rekurs auf die vom Externalismus erzwungene Modifikation der skeptischen Hypothese auch dieser Herausforderung begegnet werden? Zunächst möchte ich zugestehen, dass auch wenn uns eine auf empirischen Gründen beruhende Rechtfertigung des Einfachheitsprinzips gelingen sollte, sie in der Auseinandersetzung mit dem Skeptiker unbefriedigend bleibt. Ich gestehe außerdem $\mathrm{zu}$, dass eine apriorische Rechtfertigung des allgemeinen Prinzips der Einfachheit prima facie unmöglich scheint. Allerdings denke ich, dass man einen Spezialfall dieses Prinzips klarerweise a priori rechtfertigen kann.

Nehmen wir an die Erklärung E1 ist festgelegt auf die Annahme (x \& y), und Erklärung E2 ist festgelegt auf die Annahme (x \& y \& z). Beide Erklärungen erklären die gleichen Daten und beide erklären sie gleich gut. Die Wahrscheinlichkeit der beiden Erklärungen wird bestimmt durch die Multiplikation der Wahrscheinlichkeiten ihrer Konstituenten: $\mathrm{P}(\mathrm{E} 1)=\mathrm{P}(\mathrm{x}) \times \mathrm{P}(\mathrm{y}) ; \mathrm{P}(\mathrm{E} 2)=\mathrm{P}(\mathrm{x}) \times \mathrm{P}(\mathrm{y}) \times \mathrm{P}(\mathrm{z})$. Deswegen gilt, wenn $\mathrm{P}(\mathrm{z})<1$, dann $\mathrm{P}(\mathrm{E} 2)<\mathrm{P}(\mathrm{E} 1)$. Diese Überlegung beweist natürlich nicht generell, dass die einfachere Erklärung immer mit größerer Wahrscheinlichkeit wahr ist, aber für den fraglichen Spezialfall der Erklärungen E1 und E2 beweist sie a priori, dass die komplexere Erklärung E2 weniger wahrscheinlich ist. Der Spezialfall lässt sich folgendermaßen charakterisieren: Zwei Erklärungen E1 und E2 sind gleich erklärungskräftig. Eine der beiden Erklärungen ist um ein Konjunkt reicher als die andere, die Wahrscheinlichkeit des zusätzlichen Konjunkts ist kleiner als 1 und ohne das zusätzliche Konjunkt kommt beiden Erklärungen die gleiche Wahrscheinlichkeit zu.

Kann die Rechtfertigung dieses Spezialfalles auf die Standard-Erklärung unserer Erfahrungen (gemäß der Außenwelthypothese) und auf die skeptische Erklärung (gemäß der Hypothese SH2) übertragen werden. Die Außenwelthypothese nimmt an, dass wir seit Anbeginn unserer Existenz bis zum jetzigen Zeitpunkt in einer mit Gegenständen bevölkerten Welt leben. Die Standard-Erklärung unserer Erfahrungen E(AW), die von 
dieser Außenwelthypothese ihren Ausgang nimmt, wird zwei Suberklärungen enthalten. Eine enthält eine Menge an Behauptungen A, die Standard-Erfahrungen erklärt, und die andere enthält eine Menge an Behauptungen B, die Halluzinationen erklärt. ${ }^{13}$ Vereinfachend dargestellt hat die Standarderklärung demnach die Form:

\section{$\mathrm{E}(\mathrm{AW}): \mathrm{A} \& \mathrm{~B}$}

Die skeptische Erklärung unserer Erfahrungen E(SH2), anhand der skeptischen Hypothese SH2, nimmt dagegen an, wir seien vor fünf Minuten eingetankt worden, woraufhin die Welt, in der wir vorher lebten, mitsamt den uns bekannten Gegenständen zerstört wurde. Die Erfahrungen vor der Eintankung (Normalerfahrungen und Halluzinationen) erklärt diese Hypothese ebenso wie die Außenwelthypothese mithilfe von (A \& B), schließlich gehen wir davon aus, dass es sich dabei um die bestmögliche Erklärung handelt. Sie wird aber zusätzlich eine Menge an Behauptungen C enthalten müssen, um die Erfahrungen nach der Eintankung zu erklären (z.B. »Es gibt einen Supercomputer mit gigantischer Rechenleistung«, „Dieser Supercomputer ist mit Elektroden an die Nervenenden unseres Gehirns angeschlossen«, usw.) Die skeptische Erfahrungserklärung hat demnach die Form:

\section{$\mathrm{E}(\mathrm{SH} 2): \mathrm{A} \& \mathrm{~B} \& \mathrm{C}$}

Die Erklärung E(SH2) ist also um mindestens ein Konjunkt reicher als E(AW). Es ist weiterhin der Fall, dass die Wahrscheinlichkeit des zusätzlichen Konjunks kleiner als 1 ist - die zusätzlichen Annahmen des Skeptikers sind in keinster Weise notwendig -, und ohne das zusätzliche Konjunkt kommt beiden Erklärungen die gleiche Wahrscheinlichkeit zu, schließlich sind beide Erklärungen bis auf das zusätzliche Konjunkt C identisch. Damit kann dann genau wie beim oben diskutierten Spezialfall a priori verteidigt werden, dass

\footnotetext{
13 Welche Art von Annahmen A und B beinhalten, hängt von der Theorie von Erklärungen ab, die man bevorzugt. Gemäß dem deduktiv-nomologischen Modell wird A und B jeweils mindestens ein Naturgesetzt enthalten (s. Hempel 1965, 336-376). Demgegenüber sind Naturgesetze gemäß dem kausalen Modell von Erklärungen nicht Teil der erklärenden Information, die A und B bereit stellt (s. Lewis 1986, 239).
} 
die komplexere Erklärung E(SH2) weniger wahrscheinlich ist als die einfachere Erklärung $\mathrm{E}(\mathrm{AW})$.

Wir können daher zusammenfassend ein Argument präsentieren, das der Herausforderung (II) zu begegnen weiß:

(a) Die Erklärung E(AW), die die Außenwelthypothese für unsere Erfahrungen gibt, unterscheidet sich von der Erklärung $\mathrm{E}(\mathrm{SH} 2)$, die die modifizierte skeptische Hypothese für unsere Erfahrungen gibt, nur darin, dass E(SH2) um mindestens eine Suberklärung (d.h. um mindestens ein Konjunktionsglied) reicher ist als $\mathrm{E}(\mathrm{AW})$.

(b) Die Wahrscheinlichkeit von E(AW) ist genauso groß wie die Wahrscheinlichkeit von E(SH2) ohne das zusätzliche Konjunktionsglied.

(c) Die Wahrscheinlichkeit des zusätzlichen Konjunktionsgliedes ist kleiner 1.

(d) Dann gilt: $\mathrm{E}(\mathrm{SH} 2)$ ist weniger wahrscheinlich als $\mathrm{E}(\mathrm{AW})$ und deswegen kann $\mathrm{E}(\mathrm{SH} 2)$ begründet ausgeschlossen werden

Obwohl ich (a)-(d) für den interessantesten Versuch halte, auf die Herausforderung (II) der antiskeptischen Strategie des Schlusses auf die beste Erklärung zu reagieren, und obwohl ich (a)-(d) an anderer Stelle explizit vertreten und verteidigt habe (s. Briesen 2008), erscheint mir (a)-(d) mittlerweile eindeutig fehlerhaft. Insbesondere Prämisse (a) ist problematisch.

Wenn es wirklich richtig wäre, dass sich die skeptische Erklärung unserer Erfahrungen $\mathrm{E}(\mathrm{SH} 2)$ von der Standard-Erklärung $\mathrm{E}(\mathrm{AW})$ nur durch mindestens ein zusätzliches Konjunktionsglied unterscheiden würde, so wäre die skeptische Erklärung nichts weiter als ein Spezialfall der Standard-Erklärung. Aber das kann nicht richtig sein, schließlich sind die beiden Erklärungen doch inkompatibel zueinander, d.h. eine der Erklärungen wird sich auf irgendetwas festlegen, was die jeweils andere verneint. Und damit verlieren wir schon die Analogie zum oben angeführten Spezialfall, in dem wir leicht a priori nachweisen konnten, dass die einfachere Erklärung mit größerer 
Wahrscheinlichkeit wahr ist als die komplexe. ${ }^{14}$ Die oben angeführte Charakterisierung der beiden Erklärungen $\mathrm{E}(\mathrm{AW})$ und $\mathrm{E}(\mathrm{SH} 2)$ ist also nicht nur stark vereinfachend, sie ist auch in entscheidender Hinsicht irreführend. Wie lassen sich die beiden Erklärungen etwas präziser charakterisieren?

Um beide Erklärungen vergleichen zu können, betrachten wir sie als Erklärungen der Erfahrungen die wir zwischen zwei Zeitpunkten $t_{0}$ und $t_{2}$ machen. Zwischen diesen Zeitpunkten liegt der Zeitpunkt $t_{1}$, zu dem wir der skeptischen Hypothese SH2 gemäß eingetankt wurden. Die skeptische Erklärung E(SH2) unserer Erfahrungen hat demnach die Form:

$\mathrm{E}(\mathrm{SH} 2)$ : [Für alle Erfahrungen zwischen $\mathrm{t}_{0}$ und $\mathrm{t}_{1}$ gilt: $(\mathrm{A}$ oder B)] UND [Für alle Erfahrungen zwischen $t_{1}$ und $t_{2}$ gilt: $C$.]

Wobei die Aussagenmenge A alle Normalerfahrungen und die Aussagenmenge B alle Halluzinationen vor der Eintankung erklären. Die Aussagenmenge C hingegen erklärt alle vom Computer generierten Erfahrungen nach der Eintankung.

Demgegenüber hat die Standard-Erklärung $\mathrm{E}(\mathrm{AW})$ unserer Erfahrungen im genannten Zeitraum folgende Form:

$\mathrm{E}(\mathrm{AW})$ : [Für alle Erfahrungen zwischen $\mathrm{t}_{0}$ und $\mathrm{t}_{1}$ gilt: $(\mathrm{A}$ oder B)] UND [Für alle Erfahrungen zwischen $t_{1}$ und $t_{2}$ gilt: (A oder B).]

Weil die Standard-Erklärung nicht wie die skeptische Erklärung davon ausgeht, dass wir zu $t_{1}$ eingetankt wurden, erklärt sie alle Erfahrungen im Zeitraum $t_{1}-t_{2}$ genauso wie die Erfahrungen im Zeitraum $t_{0}-t_{1}$.

Anhand dieser adäquateren Charakterisierung der beiden zur Debatte stehenden Erklärungen ist nicht nur einsichtig, dass sich beide Erklärungen widersprechen, es ist darüber hinaus auch klar, dass Prämisse (a) unseres Arguments (a)-(d) falsch ist. Es ist

\footnotetext{
${ }^{14}$ Dieses Problem habe ich in einem früheren Aufsatz bereits erkannt, habe dort aber dafür argumentiert, dass es sich in einer Weise lösen lässt, die mit der angeführten Idee auf Herausforderung (II) zu reagieren, verträglich ist (s. Briesen 2008, 20).
} 
nicht der Fall, dass sich die Erklärung E(AW) von der Erklärung E(SH2) nur darin unterscheidet, dass $\mathrm{E}(\mathrm{SH} 2)$ um mindestens ein Konjunktionsglied reicher ist als $\mathrm{E}(\mathrm{AW})$. Das Argument (a)-(d) kann also nicht dazu dienen, der Herauforderung (II) zu begegnen. Die antiskeptische Strategie des Schlusses auf die beste Erklärung krankt (auch in der Kombination mit der Strategie des semantischen Externalismus) weiterhin daran, dass auf besagte Herausforderung nicht angemessen reagiert werden kann.

\section{Schlussbemerkung}

Es hat sich ergeben, dass der semantische Externalismus den Skeptiker unter Druck setzt. Falls externalistische Kernthesen zutreffen, ist der Skeptiker gezwungen, den wichtigsten Baustein seines Arguments, die skeptische Hypothese, umzuformulieren. Die vom Externalismus erzwungene reformulierte Hypothese geht davon aus, dass wir ein erst kürzlich eingetanktes Gehirn sind. Das skeptische Argument ist durch diese Modifikation in seiner Durchschlagskraft eingeschränkt. Vor allem für Überzeugungen, die sich auf unsere Vergangenheit beziehen, kann das Argument nicht mehr beweisen, dass die fragliche Überzeugung kein Fall von Wissen ist. Diese Einschränkung hat sich aber in skeptischer Hinsicht als unproblematisch erwiesen. Erstens ist das modifizierte Argument immer noch bedrohlich genug (s. 6.1). Und zweitens kann auch das modifizierte Argument nicht durch Erinnerungen und die darauf fußenden Überzeugungen blockiert werden (s. 6.2).

Allerdings ist die Erklärung, die die modifizierte Hypothese für unsere sinnlichen

Erfahrungen geben kann, in bestimmter Hinsicht eindeutig komplexer als die Standarderklärung unserer Erfahrung. Damit ist durch die vom Externalismus erzwungene Modifikation eine Schwierigkeit der etwas in Vergessenheit geratenen antiskeptischen Strategie des Schlusses auf die beste Erklärung behoben. Doch die zweite zentrale Schwierigkeit der Strategie des Schlusses auf die beste Erklärung, die darin besteht, zu verteidigen, warum wir überhaupt epistemisch gerechtfertigt sind, eine einfache Erklärung einer komplexeren vorzuziehen, bleibt bestehen. Sie kann nicht einfach auf der Basis der 
vom Externalismus erzwungenen Modifikation der skeptischen Hypothese behoben werden (s. 6.2). Auch durch eine Verbindung der beiden antiskeptischen Strategien des semantischen Externalismus und des Schlusses auf die beste Erklärung lässt sich die philosophische Skepsis also nicht befriedigend zurückweisen. Die antiskeptische Überzeugungskraft des semantischen Externalismus ist und bleibt empfindlich eingeschränkt. ${ }^{15}$

\section{Literatur}

Boghossian, Paul 1989: Content and Self-Knowledge, in: Philosophical Topics 17, 5-26. Briesen, Jochen 2008: Scepticism, Externalism, and Inference to the Best Explanation, in Abstracta 4, 5-26.

Chalmers, David 2005: The Matrix as Metaphysics, in: C. Grau (Hg.), Philosophers Explore the Matrix, Oxford, 132-176.

Hawthorne, John 2003: The Case for Closure, in: M. Steup and E. Sosa (Hg.), Contemporary Debates in Epistemology, Oxford, 26-43.

Hempel, Carl 1965: Aspects of Scientific Explanation and Other Essays in the Philosophy of Science, New York.

Lewis, David 1986: Causal Explanation, in: D. Lewis, Philosophical Papers, Volume II, Oxford, 214-240.

Lipton, Peter 1991: Inference to the best Explanation, London/New York.

Müller, Olaf 2003: Wirklichkeit ohne Illusionen, Bd. I, Paderborn.

Pryor, James 2000: The Skeptic and the Dogmatist, in: Nous 34, 517-549.

Putnam, Hilary 1975: The Meaning of Meaning, in: H. Putnam, Mind, Language and Reality, Philosophical Papers, Volume II, Cambridge, 215-71.

Putnam, Hilary 1981: Reason, Truth and History, Cambridge.

\footnotetext{
${ }^{15}$ Für hilfreiche Diskussionen, kritische Anmerkungen und sonstige Unterstützung danke ich herzlich Elke Brendel, David Chalmers, Dina Emundts, Thomas Grundmann, Rolf-Peter Horstmann, Torfinn Huvenes, Olaf Müller, Dominik Perler, Martin Smith, Clas Weber und einem anonymen Gutachter der Zeitschrift für philosophische Forschung.
} 
Vogel, Jonathan 1990: Cartesian Skepticism and Inference to the best Explanation, in: Journal of Philosophy 87, 658-666.

Williamson, Timothy 2000: Knowledge and its Limits, Oxford. 